\title{
Novel Trans-Spanned Palladium Complexes as Efficient Catalysts in Mild and Amine-Free Cyanation of Aryl Bromides under Air
}

\author{
Olga Grossman and Dmitri Gelman* \\ dgelman@chem.ch.huji.ac.il
}

General Considerations. Palladium acetate (98\%), potassium phosphate, N,N-dimethylformamide, potassium ferrocyanide, potassium ferricyanide and all starting aryl halides were purchased from Aldrich. All reagents were used without further purification. 1,8-dibromotryptycene was synthesized according the previously published procedure. ${ }^{1}$ All reagents were weighed and handled in air. Flash column chromatography was performed with Merck ultra pure silica gel (230-400 mesh) purchased from Fluka. All reactions were carried out under an air atmosphere in oven-dried glassware. ${ }^{1} \mathrm{H}-\mathrm{NMR}$ and ${ }^{13} \mathrm{C}-\mathrm{NMR}$ spectra were recorded on a Bruker $300 \mathrm{MHz}$ instrument with chemical shifts reported in ppm relative to the residual deuterated solvent or the internal standard tetramethylsilane. Gas chromatography analyses were performed on a Hewlett Packard 5890 instrument with a FID detector and a Hewlett Packard 25 m x 0.2 mm i.d. Supelcowax-10 capillary column. Yields refer to isolated yields of compounds greater than $95 \%$ purity as determined proton Nuclear Magnetic Resonance spectroscopy ( ${ }^{1} \mathrm{H}-\mathrm{NMR}$ ) analysis. Yields reported in Table 2 are an average of two runs. The CAS numbers of the known compound were listed. Spectroscopy data of the known compounds matches with the data reported in the corresponding references.

1,8-dibromotriptycene. ${ }^{1}$ 1,8-dibromoanthracene (10 g, $\left.29 \mathrm{mmol}\right)$ in $75 \mathrm{~mL}$ of 1,2-dimethoxyethane and isopenthyl nitrite $(5.75 \mathrm{~g}, 50 \mathrm{mmol})$ were placed in a $500 \mathrm{~mL}$ three-necked round-bottomed flask fitted with a reflux condenser and a mechanical stirrer. The mixture was heated to reflux and the solution of anthranilic acid $(8.5 \mathrm{~g}, 60 \mathrm{mmol})$ in $40 \mathrm{~mL}$ of 1,2-dimethoxyethane was added slowly over 40 min by means of a syringe pump. The mixture was cooled down to room temperature and an additional portion of isopenthyl nitrite $(5.75 \mathrm{~g}, 50 \mathrm{mmol})$ was added at once. After resuming reflux, another portion of anthranilic acid $(8.5 \mathrm{~g}, 60 \mathrm{mmol})$ in $40 \mathrm{~mL}$ of 1,2-dimethoxyethane was added over $30 \mathrm{~min}$. The mixture was cooled down to room temperature and $30 \mathrm{~mL}$ of methanol along with $250 \mathrm{~mL}$ of $10 \%$ sodium hydroxide were added. The brown suspension was cooled to ca. $10{ }^{\circ} \mathrm{C}$ and filtered. The residue was washed with 3 portions of chilled methanol/water (4/1). The crude product and $5 \mathrm{~g}$ of maleic anhydride were transferred to a 250 round-bottomed flask and dissolved in $50 \mathrm{~mL}$ of triethyleneglycol dimethyl ether. The resulting solution was heated under reflux for $15 \mathrm{~min}$, cooled and worked-up with $10 \%$ sodium hydroxide solution as was described previously. The product was isolated by filtration and thorough washing with methanol/water affording 8-9 g (66-75\% yield) of $95 \%$ pure material. ${ }^{1} \mathrm{H}$ NMR $\left(300 \mathrm{MHz}, \mathrm{CDCl}_{3}\right): 7.55$ (m, 1H), $7.43(\mathrm{~m}, 1 \mathrm{H}), 7.33$ (d, J = 7.2 Hz, 2H), 7.23 (d, J = 7.2 Hz, $2 \mathrm{H}), 7.08-7.06(\mathrm{~m}, 2 \mathrm{H}), 6.88$ (t, J = 9 $\mathrm{Hz}, 2 \mathrm{H}), 6.44(\mathrm{~s}, 1 \mathrm{H}), 5.46(\mathrm{~s}, 1 \mathrm{H}) .{ }^{13} \mathrm{C} \mathrm{NMR}\left(75 \mathrm{MHz}, \mathrm{CDCl}_{3}\right): \delta 147.54,144.77,144.00,143.20,129.02$, $126.86,126.54,125.80,124.59,123.72,122.68,119.54,54.72,52.30$. 
1,8-bis(diisopropylphosphino)triptycene (L1). To a cold stirred solution $\left(-78^{\circ} \mathrm{C}\right)$ of $1,8-$ dibromotriptycene ( $3 \mathrm{~g}, 7.28 \mathrm{mmol})$ and TMEDA $(5.5 \mathrm{~mL}, 5 \mathrm{eq})$ in dry THF (35 mL) n-BuLi (1.6 M, 9.2 $\mathrm{mL}, 2 \mathrm{eq}$ ) was added over a period of 30-35 min. The resulting yellow to brown solution was stirred for additional $15 \mathrm{~min}$ and diisopropylchlorophosphine ( $2.3 \mathrm{~mL}, 14.5 \mathrm{mmol}, 2 \mathrm{eq})$ solution in THF ( $3 \mathrm{~mL})$ was added dropwise. The nearly colorless solution was allowed to reach room temperature and then refluxed for 1 hour. After being cooled, ethyl acetate $(50 \mathrm{~mL})$ was added; the organic phase was successively washed with sodium bicarbonate and water, dried and evaporated. The crude material was purified by filtration through a pad of silica gel and crystallized from methanol affording $1.8 \mathrm{~g}$ (51\% yield) of analytically pure material. ${ }^{1} \mathrm{H}$ NMR $\left(\right.$ THF-d $\left._{8}\right): 7.51(\mathrm{t}, \mathrm{J}=5 \mathrm{~Hz}, 1 \mathrm{H}), 7.35-7.30(\mathrm{~m}, 4 \mathrm{H}), 7.12(\mathrm{~d}, \mathrm{~J}=7.5 \mathrm{~Hz}, 2 \mathrm{H}), 6.94(\mathrm{t}, \mathrm{J}=$ $7.5 \mathrm{~Hz}, 2 \mathrm{H}), 6.90-6.87(\mathrm{~m}, 2 \mathrm{H}), 5.44(\mathrm{~s}, 1 \mathrm{H}), 2.23-2.18(\mathrm{~m}, 2 \mathrm{H}), 2.07-2.01(\mathrm{~m}, 2 \mathrm{H}), 1.21$ (dd, J = $7 \mathrm{~Hz}, \mathrm{~J}=$ $7 \mathrm{~Hz}, 6 \mathrm{H}), 1.06(\mathrm{dd}, \mathrm{J}=8 \mathrm{~Hz}, \mathrm{~J}=8 \mathrm{~Hz}, 6 \mathrm{H}), 0.92(\mathrm{dd}, \mathrm{J}=7 \mathrm{~Hz}, \mathrm{~J}=7 \mathrm{~Hz}, 6 \mathrm{H}), 0.75(\mathrm{dd}, \mathrm{J}=7 \mathrm{~Hz}, \mathrm{~J}=3 \mathrm{~Hz}$, $6 \mathrm{H}) .{ }^{31} \mathrm{P}$ NMR (THF-d 8 ): $\delta$ 4.75. Anal. Calcd. for $\mathrm{C}_{32} \mathrm{H}_{40} \mathrm{P}_{2}$ : C 78.98; H 8.29. Found: C 79.26; H 8.22.

Representative procedure for palladium-catalyzed cyanation of aryl bromides. An oven-dried singleuse screw-capped tube was charged with $\mathrm{Pd}(\mathrm{OAc})_{2}(2.2 \mathrm{mg}, 0.01 \mathrm{mmol}, 1 \mathrm{~mol} \%), \mathbf{L 1}(7.3 \mathrm{mg}, 0.015$ mmol, $1.5 \mathrm{~mol} \%), \mathrm{K}_{4} \mathrm{Fe}(\mathrm{CN})_{6}(112 \mathrm{mg}, 0.3 \mathrm{mmol}, 0.3 \mathrm{eq})$ and $\mathrm{K}_{3} \mathrm{PO}_{4}(430 \mathrm{mg}, 2 \mathrm{mmol}, 2 \mathrm{eq})$ followed by anhydrous DMF (4 mL). The yellow suspension was stirred for 5-10 min. Then the 1-bromonaphthalene (207 mg, $1 \mathrm{mmol}, 1 \mathrm{eq}$ ) was injected at once. The tube was sealed and the reaction mixture was stirred at $90{ }^{\circ} \mathrm{C}$ for 24 hours. The resulting suspension was allowed to reach room temperature, diluted with water (2 $\mathrm{mL})$ and extracted with dichloromethane $(4 \mathrm{~mL})$. The combined organic phases were dried over MgSO4, concentrated, and the residue was purified by flash chromatography on silica with ethyl acetate/hexane to provide the desired product in $98 \%$ yield and $95 \%$ purity $(149 \mathrm{mg})$.

\section{Analytical data}<smiles>COC(=O)c1ccc(C#N)cc1</smiles>

Methyl-4-cyanobenzoate (CAS Registry Number: 1129-35-7) $)^{2}:{ }^{1} \mathrm{H}$ NMR $\left(300 \mathrm{MHz}, \mathrm{CDCl}_{3}\right): \delta=8.15$ $(2 \mathrm{H}, \mathrm{d}, J=8.56), 7.75(2 \mathrm{H}, \mathrm{d}, J=8.56), 3.97 \mathrm{ppm}(3 \mathrm{H}, \mathrm{s}) ;{ }^{13} \mathrm{C}$ NMR $\left(300 \mathrm{MHz}, \mathrm{CDCl}_{3}\right): \delta 165.4,133.9$, 132.2, 130.1, 117.9, 116.3, 52.7; IR $\left(\mathrm{CHCl}_{3}\right): v=2225 \mathrm{~cm}^{-1}$; MS $(70 \mathrm{eV}, \mathrm{EL}): \mathrm{m} / z: 161\left[\mathrm{M}^{+}\right], 130\left[\mathrm{M}^{+}-\right.$ $\mathrm{OCH}_{3}$.

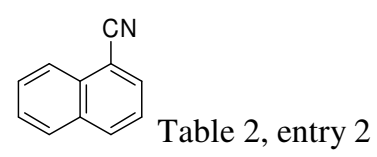

1-naphtonitrile (CAS Registry Number: 86-53-3) ${ }^{11}:{ }^{1} \mathrm{H}$ NMR $\left(300 \mathrm{MHz}, \mathrm{CDCl}_{3}\right): \delta=8.24(1 \mathrm{H}, \mathrm{d}, J=8.5)$, $8.09(1 \mathrm{H}, \mathrm{d}, J=8.5), 8.0-7.87(2 \mathrm{H}, \mathrm{m}, \mathrm{ar}), 7.75-7.58(2 \mathrm{H}, \mathrm{m}, \mathrm{ar}), 7.58-7.49(1 \mathrm{H}, \mathrm{m}, \mathrm{ar}) ;{ }^{13} \mathrm{C} \mathrm{NMR}(300$ $\left.\mathrm{MHz}, \mathrm{CDCl}_{3}\right): \delta(\mathrm{ppm})=133.27,132.88,132.63,132.32,128.63,128.58,127.53,125.13,124.91,117.76$, 110.14; IR $\left(\mathrm{CHCl}_{3}\right): v=2216 \mathrm{~cm}^{-1}$; MS $(70 \mathrm{eV}, \mathrm{EL}): \mathrm{m} / z: 153\left[\mathrm{M}^{+}\right]$. 


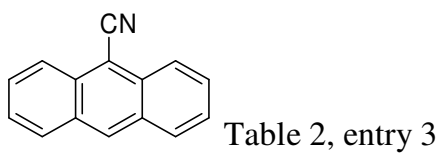

Anthracene-10-carbonitrile (CAS Registry Number: 1210-12-4) ${ }^{9}:{ }^{1} \mathrm{H}$ NMR $\left(300 \mathrm{MHz}, \mathrm{CDCl}_{3}\right): \delta=8.69$ $(1 \mathrm{H}, \mathrm{s}), 8.43(2 \mathrm{H}, \mathrm{d}, J=8.7), 8.09(2 \mathrm{H}, \mathrm{d}, J=8.7), 7.73(2 \mathrm{H}, \mathrm{t}, J=7.6), 7.6 \mathrm{ppm}(2 \mathrm{H}, \mathrm{t}, J=7.65) ;{ }^{13} \mathrm{C}$ NMR $\left(300 \mathrm{MHz}, \mathrm{CDCl}_{3}\right): \delta(\mathrm{ppm})=134.04,133.17,132.67,130.48,128.89,127.12,126.28,125.16,117.22$, 105.26; IR (KBr): $v=2211 \mathrm{~cm}^{-1} ; \mathrm{mp} 173-177{ }^{0} \mathrm{C} ;{ }^{10} \mathrm{MS}(70 \mathrm{eV}, \mathrm{EL}): \mathrm{m} / z: 203\left[\mathrm{M}^{+}\right]$.<smiles>CC(C)(C)c1ccc(C#N)cc1</smiles>

4-tertbutylbenzonitrile (CAS Registry Number: 4210-32-6) ${ }^{3}:{ }^{1} \mathrm{H} \mathrm{NMR}\left(300 \mathrm{MHz}, \mathrm{CDCl}_{3}\right): \delta(\mathrm{ppm})=$ $7.57(2 \mathrm{H}, \mathrm{d}, J=8), 7.47(2 \mathrm{H}, \mathrm{d}, J=8), 1.31 \mathrm{ppm}(9 \mathrm{H}, \mathrm{s}) ;{ }^{13} \mathrm{C} \mathrm{NMR}\left(300 \mathrm{MHz}, \mathrm{CDCl}_{3}\right): \delta(\mathrm{ppm})=156.5$, 131.9, 126.1, 119.1, 109.2, 35.2, 30.9; IR $\left(\mathrm{CHCl}_{3}\right): v=2225 \mathrm{~cm}^{-1}$; MS $(70 \mathrm{eV}, \mathrm{EL}): \mathrm{m} / z: 159\left[\mathrm{M}^{+}\right], 144\left[\mathrm{M}^{+}\right.$ $\left.-\mathrm{CH}_{3}\right], 116\left[\mathrm{M}^{+}\right]$.

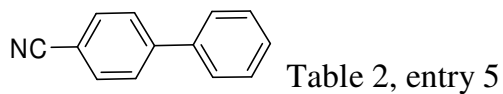

4-phenylbenzonitrile (CAS Registry Number: $2920-38-9){ }^{13}:{ }^{1} \mathrm{H} \mathrm{NMR}\left(300 \mathrm{MHz}, \mathrm{CDCl}_{3}\right): \delta=7.73-7.69$ $\left(4 \mathrm{H}, \mathrm{m}\right.$, aromatic) 7.62-7.58 $\left(2 \mathrm{H}, \mathrm{m}\right.$, aromatic) $7.50-7.46 \mathrm{ppm}\left(3 \mathrm{H}, \mathrm{m}\right.$, aromatic); ${ }^{13} \mathrm{C} \mathrm{NMR}(300 \mathrm{MHz}$, $\left.\mathrm{CDCl}_{3}\right): \delta(\mathrm{ppm})=145.64,139.14,132.57,129.09,128.64,127.71,127.21,118.94,110.87 ; \mathrm{IR}(\mathrm{KBr}): \mathrm{v}=$ $2230 \mathrm{~cm}^{-1} ; \mathrm{mp} 84-88{ }^{0} \mathrm{C} ;{ }^{14} \mathrm{MS}(70 \mathrm{eV}, \mathrm{EL}): \mathrm{m} / z: 179\left[\mathrm{M}^{+}\right]$.

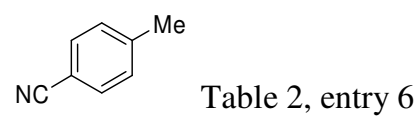

4-methylbenzonitrile (CAS Registry Number: 104-85-8) ${ }^{6}:{ }^{1} \mathrm{H}$ NMR $\left(300 \mathrm{MHz}, \mathrm{CDCl}_{3}\right): \delta=7.53(2 \mathrm{H}, \mathrm{d}$, $J=8.18), 7.27(2 \mathrm{H}, \mathrm{d}, J=8.18), 2.42 \mathrm{ppm}(3 \mathrm{H}, \mathrm{s}) ;{ }^{13} \mathrm{C} \mathrm{NMR}\left(300 \mathrm{MHz}, \mathrm{CDCl}_{3}\right): \delta(\mathrm{ppm})=143.7,132.0$, 129.8, 119.1, 109.2, 21.8; IR $\left(\mathrm{CHCl}_{3}\right): v=2234 \mathrm{~cm}^{-1}$; MS (70 eV, EL): $m / z: 117\left[\mathrm{M}^{+}\right]$.

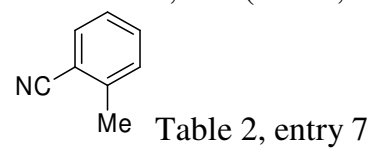

2-methylbenzonitrile (CAS Registry Number: 529-19-1) $)^{12}:{ }^{1} \mathrm{H}$ NMR $\left(300 \mathrm{MHz}, \mathrm{CDCl}_{3}\right): \delta=7.58(1 \mathrm{H}, \mathrm{d}$, $J=7.7), 7.47(1 \mathrm{H}, \mathrm{d}, J=7.2), 7.32-7.26(2 \mathrm{H}, \mathrm{m}, \mathrm{ar}) ;{ }^{13} \mathrm{C} \mathrm{NMR}\left(300 \mathrm{MHz}, \mathrm{CDCl}_{3}\right): \delta(\mathrm{ppm})=141.89,132.61$, 132.46, 130.19, 126.18, 118.13, 112.70, 20.44; IR $\left(\mathrm{CHCl}_{3}\right): v=2226 \mathrm{~cm}^{-1}$; MS (70 eV, EL): $\mathrm{m} / z: 117\left[\mathrm{M}^{+}\right]$.

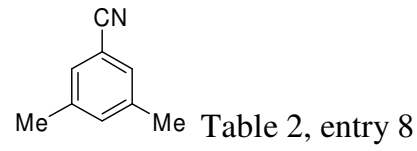

3,5-dimethylbenzonitrile (CAS Registry Number: 22445-42-7 $)^{8}:{ }^{1} \mathrm{H}$ NMR $\left(300 \mathrm{MHz}, \mathrm{CDCl}_{3}\right): \delta=7.25$ $(2 \mathrm{H}, \mathrm{s}), 7.21(1 \mathrm{H}, \mathrm{s}) 2.34 \mathrm{ppm}(6 \mathrm{H}, \mathrm{s}) ;{ }^{13} \mathrm{C} \mathrm{NMR}\left(300 \mathrm{MHz}, \mathrm{CDCl}_{3}\right): \delta(\mathrm{ppm})=138.93,134.52,129.56$, 119.14, 111.91, 20.94; MS (70 eV, EL): $m / z: 131\left[\mathrm{M}^{+}\right], 116\left[\mathrm{M}^{+}-\mathrm{CH}_{3}\right]$. 


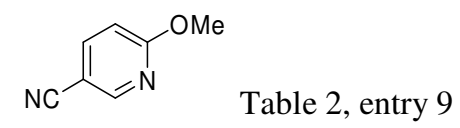

6-methoxypyridine 3-carbonitrile (CAS Registry Number: 15871-85-9) ${ }^{7}:{ }^{1} \mathrm{H}$ NMR $\left(300 \mathrm{MHz}, \mathrm{CDCl}_{3}\right)$ : $\delta=8.50(1 \mathrm{H}, \mathrm{s}), 7.76\left(1 \mathrm{H}, \mathrm{dd}, J^{1}=8.7, J^{2}=2.4\right), \quad 6.83(1 \mathrm{H}, \mathrm{d}, J=8.9), 3.98(3 \mathrm{H}, \mathrm{s}) ;{ }^{13} \mathrm{C} \mathrm{NMR}(300 \mathrm{MHz}$, $\left.\mathrm{CDCl}_{3}\right): \delta(\mathrm{ppm})=165.96,151.93,140.8,117.24,111.78,102.32,54.21 ; \mathrm{IR}(\mathrm{KBr}): \mathrm{v}=2225 \mathrm{~cm}^{-1} ; \mathrm{mp} 93-$ $97{ }^{0} \mathrm{C} ;{ }^{7} \mathrm{MS}(70 \mathrm{eV}, \mathrm{EL}): m / z: 134\left[\mathrm{M}^{+}\right], 104\left[\mathrm{M}^{+}-\mathrm{OCH}_{3}\right]$.

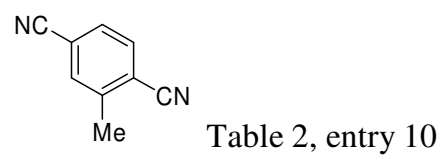

1,3-dicyano-2-methylbenzene (CAS Registry Number: 2317-22-8) ${ }^{4}{ }^{1} \mathrm{H}$ NMR $\quad\left(300 \mathrm{MHz}, \mathrm{CDCl}_{3}\right): \delta$ $(\mathrm{ppm})=7.74-7.36\left(3 \mathrm{H}, \mathrm{m}\right.$, aromatic) $2.61 \mathrm{ppm}(3 \mathrm{H}, \mathrm{s}) ;{ }^{13} \mathrm{C} \mathrm{NMR}\left(300 \mathrm{MHz}, \mathrm{CDCl}_{3}\right): \delta(\mathrm{ppm})=143.2$, 133.6, 133.1, 129.7, 117.2, 20.4; IR (KBr): $v=2226 \mathrm{~cm}^{-1}$; mp 142-145 ${ }^{0} \mathrm{C}^{5}{ }^{5} \mathrm{MS}(70 \mathrm{eV}, \mathrm{EL}): \mathrm{m} / z: 142\left[\mathrm{M}^{+}\right]$, $116\left[\mathrm{M}^{+}-\mathrm{CN}\right]$.

References:

1. Grossman, O.; Azerraf, C.; Gelman, D. Organometallics 2006, 25, 375.

2. Weissman, S. A.; Zewge, D.; Chen. C. J. Org. Chem., 2005, 70, 1508.

3. Kurihara, T. Chem\&Pharm. Bull., 1986, 34, 2747.

4. Skeean, R., W. Synthesis 1990, 628.

5. Mao, Y.; Virgil Boekelheide, V. J. Org. Chem., 1980, 45, 2746.

6. Yang, C. Org. Lett. 2004, 6, 2837.

7. Naidu, B. N.; Sorenson, M. E. Org. Lett., 2005, 7, 1391.

8. Zanon, J.; Klapars, A.; Buchwald, S. L. J. Am. Chem. Soc., 2003, 125, 2890.

9. Hughes T. V.; Cava, M. P. J. Org. Chem., 1998, 64, 313.

10. Gore, P. H.; Kamounah, F. S.; Miri, A. Y. Tetrahedron 1979, 35, 2927.

11. Stazi, F.; Palmisano, G.; Turconib, M.; Santagostino, M. Tetrahedron Lett., 2005, 46, 1815.

12. Schareina, T.; Zapf, A.; Beller, M. J. Organometallic Chem., 2004, 689, 4576.

13. Su, W.; Urgaonkar, S.; McLaughlin, P. A.; Verkade J. G. J. Am. Chem. Soc., 2004, 126, 16433.

14. Tao, B.; Boykin, D. W. J. Org. Chem., 2004, 69, 4330. 

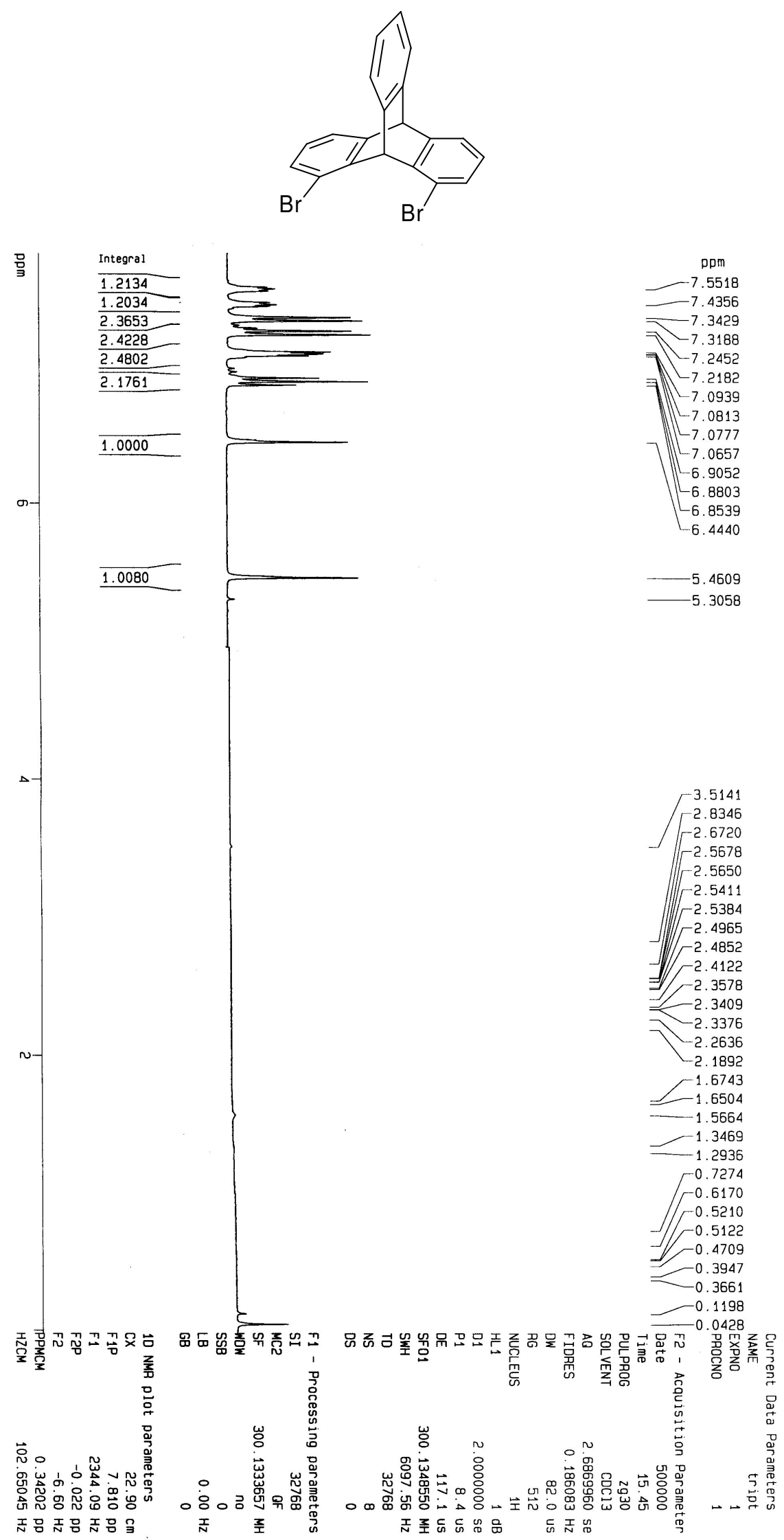


$$
E
$$



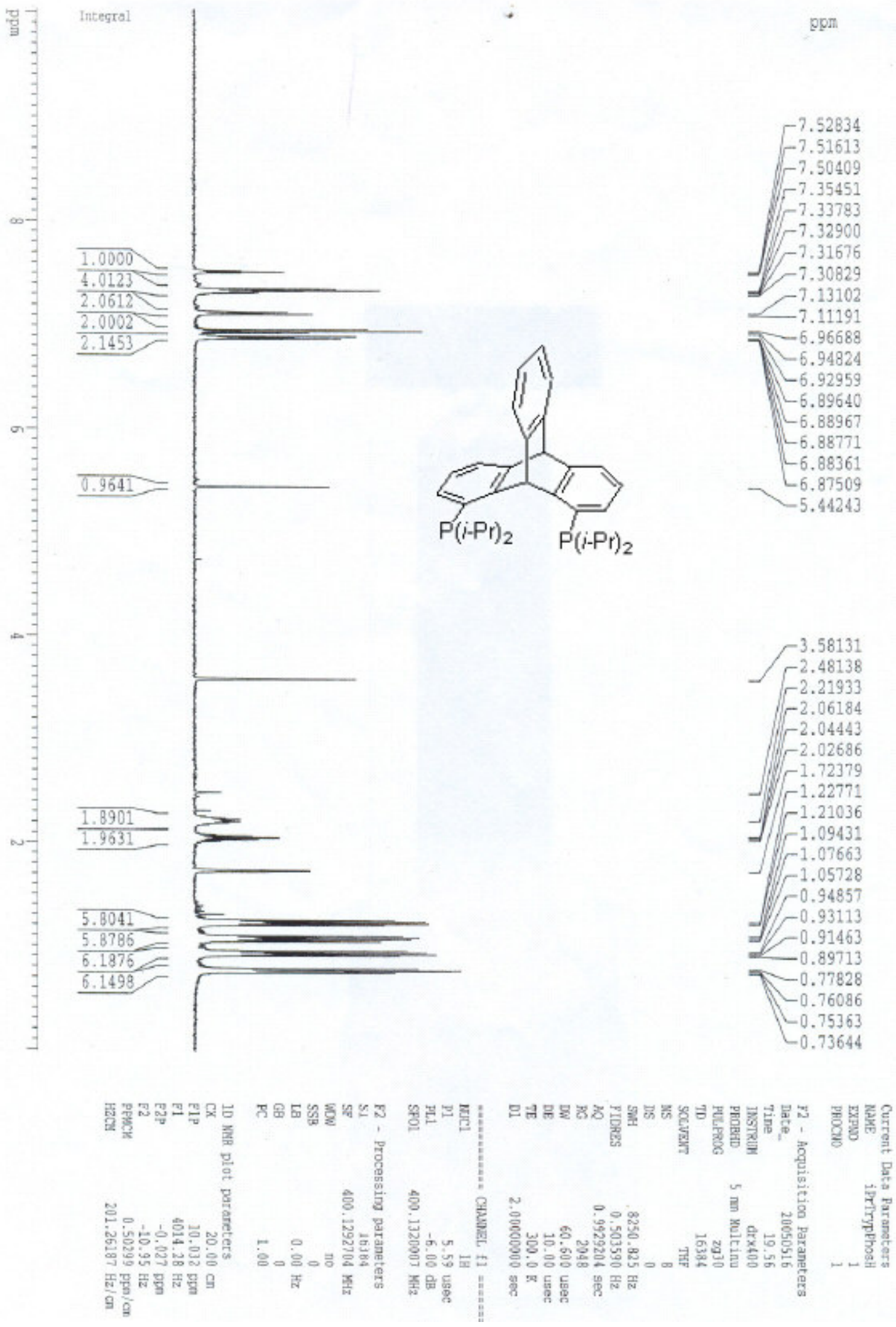


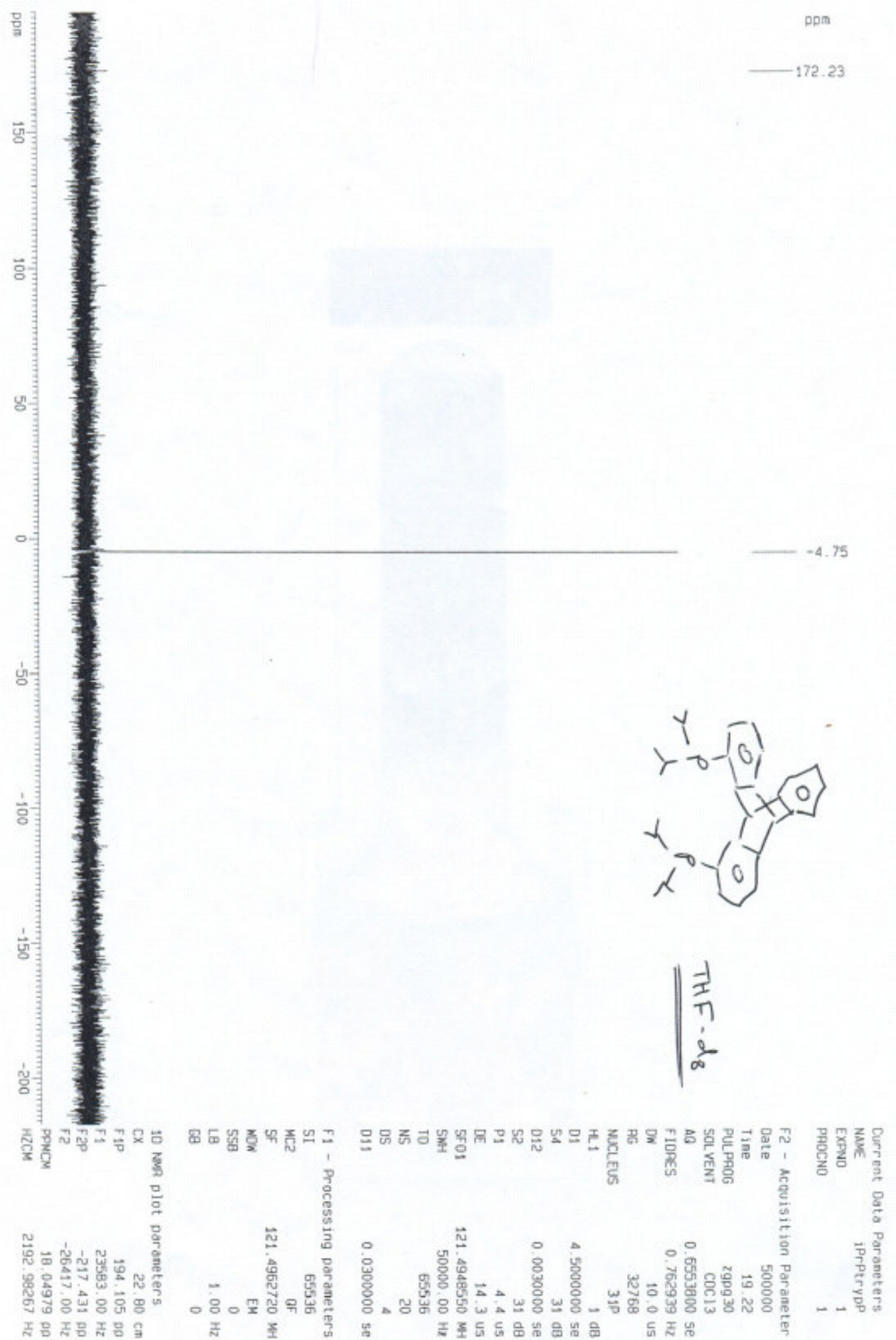




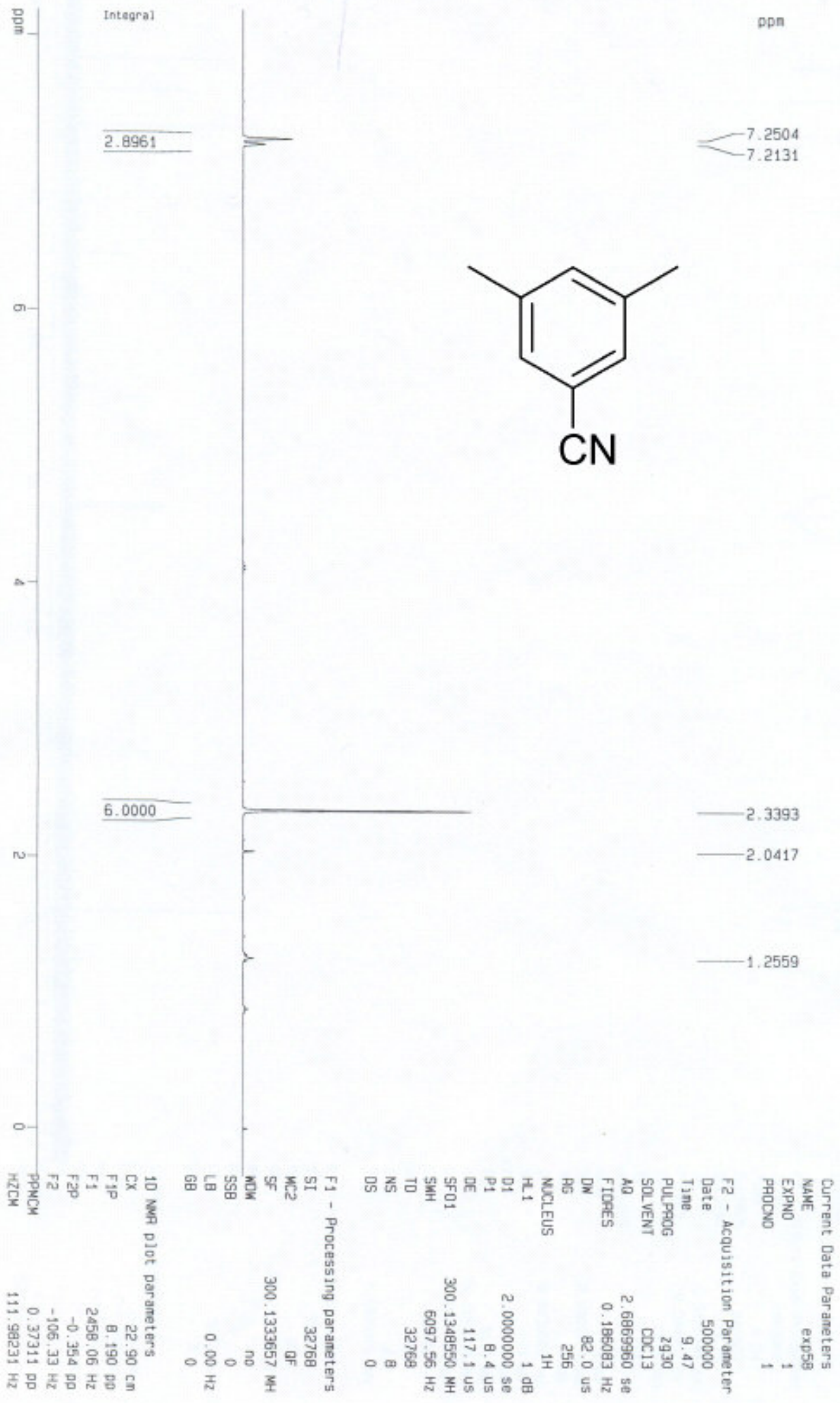




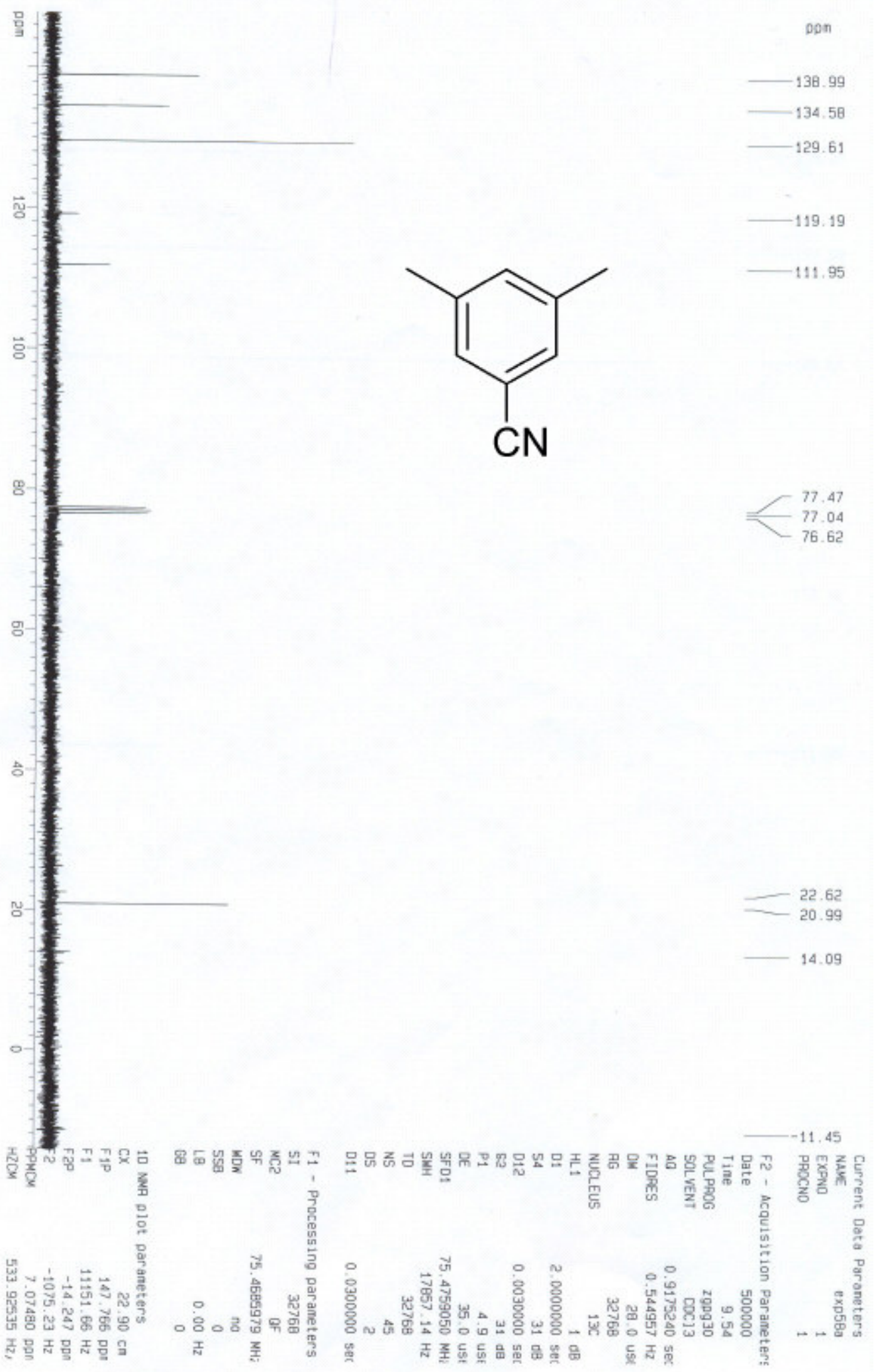




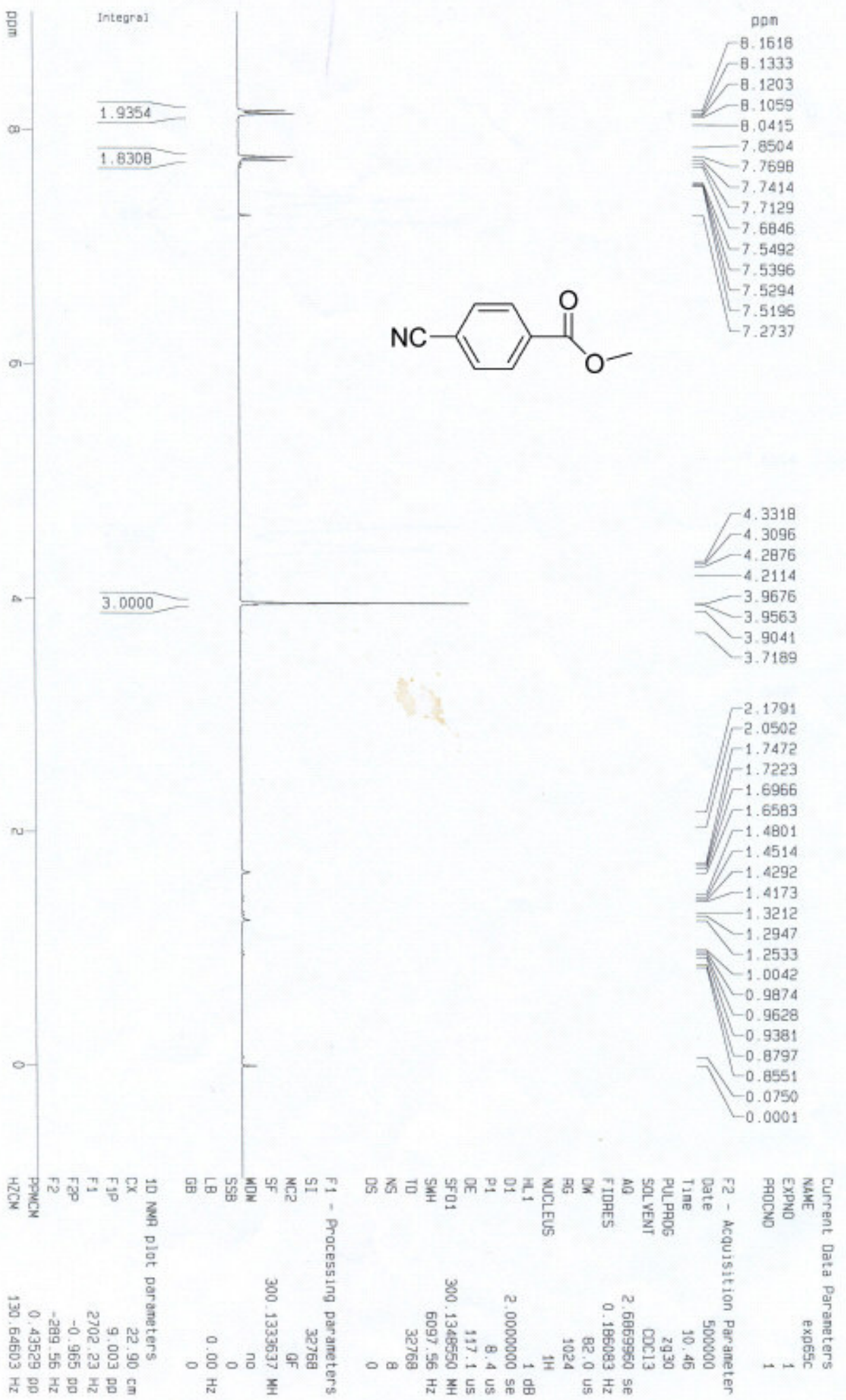




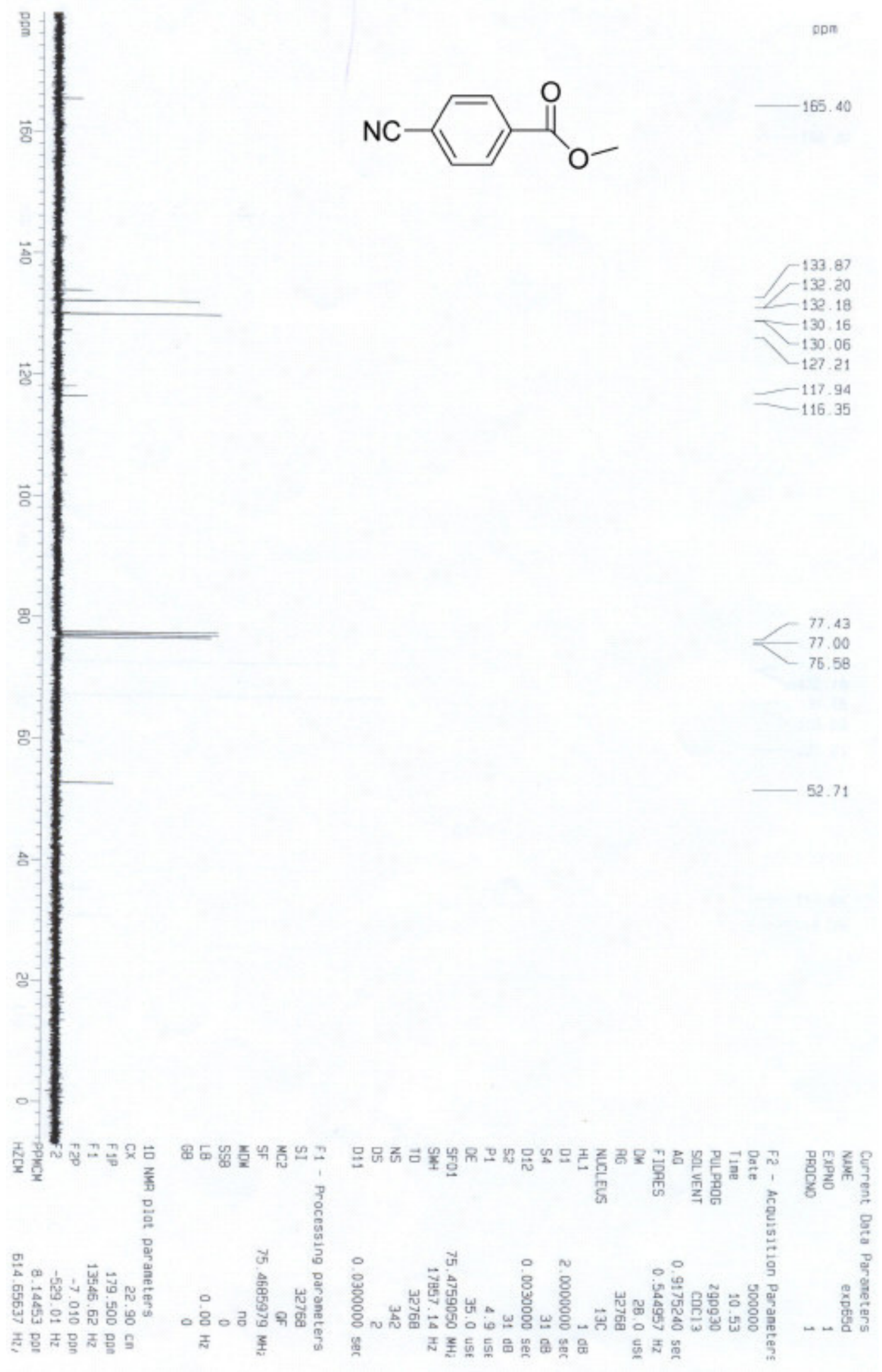




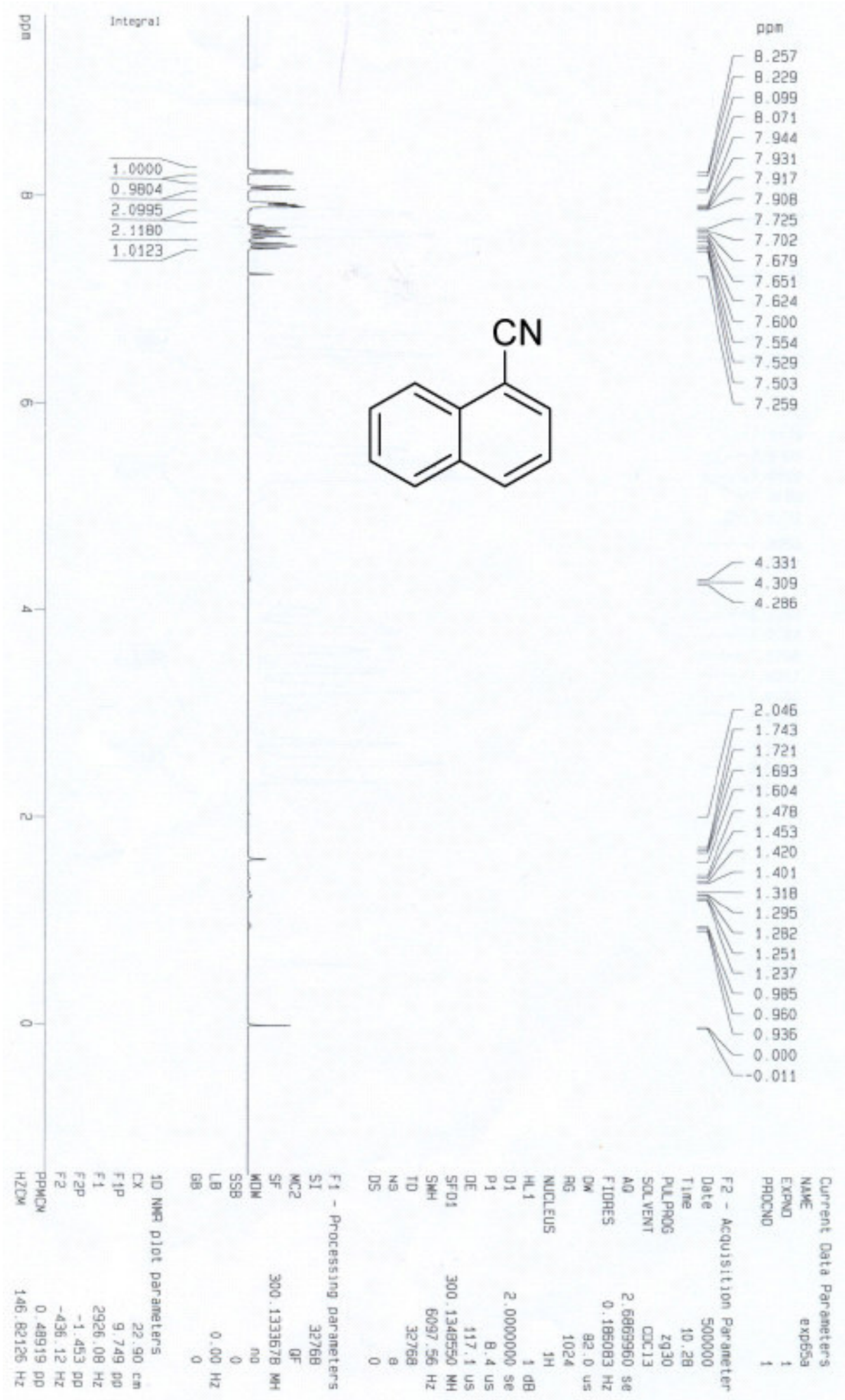




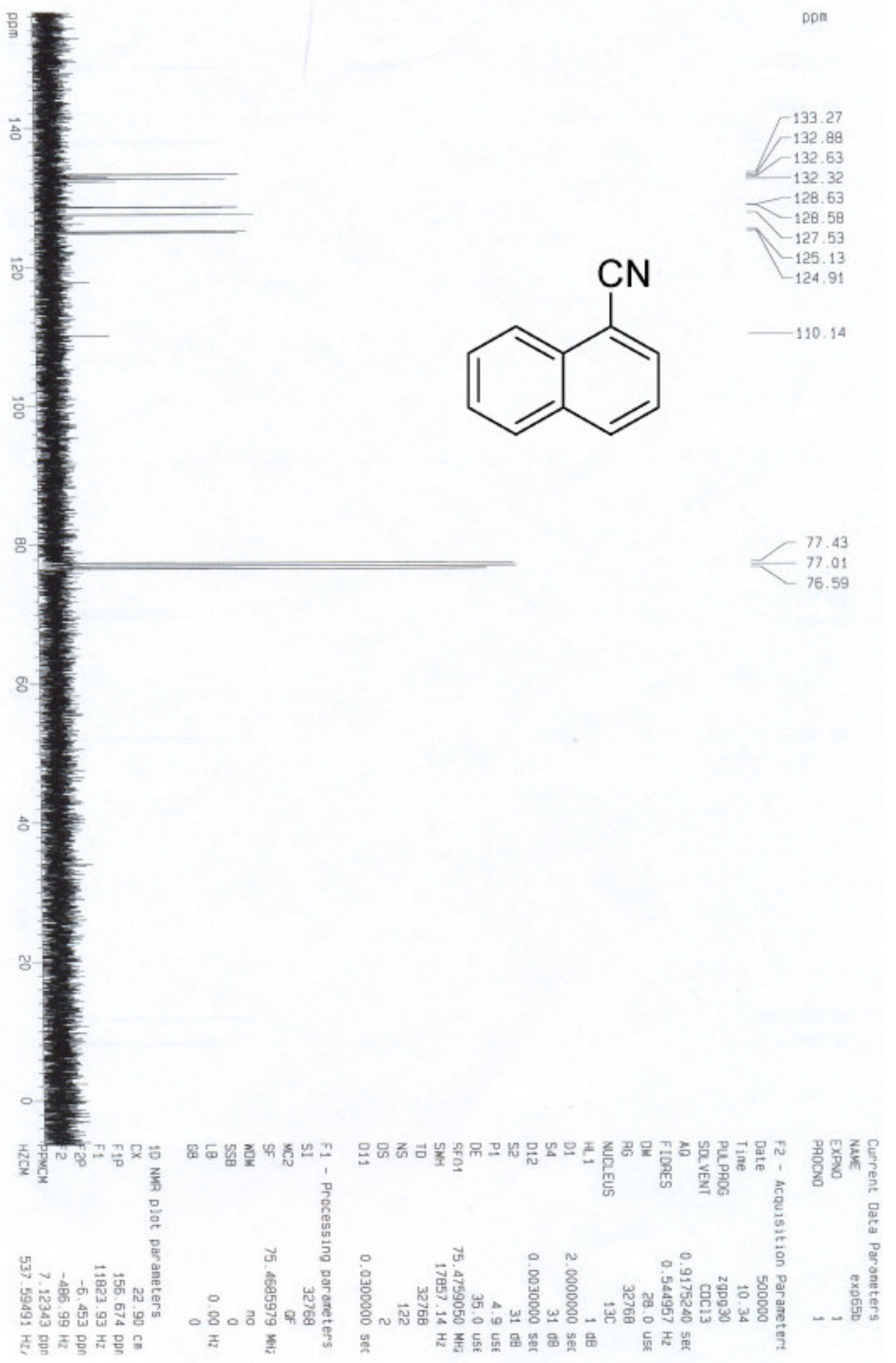




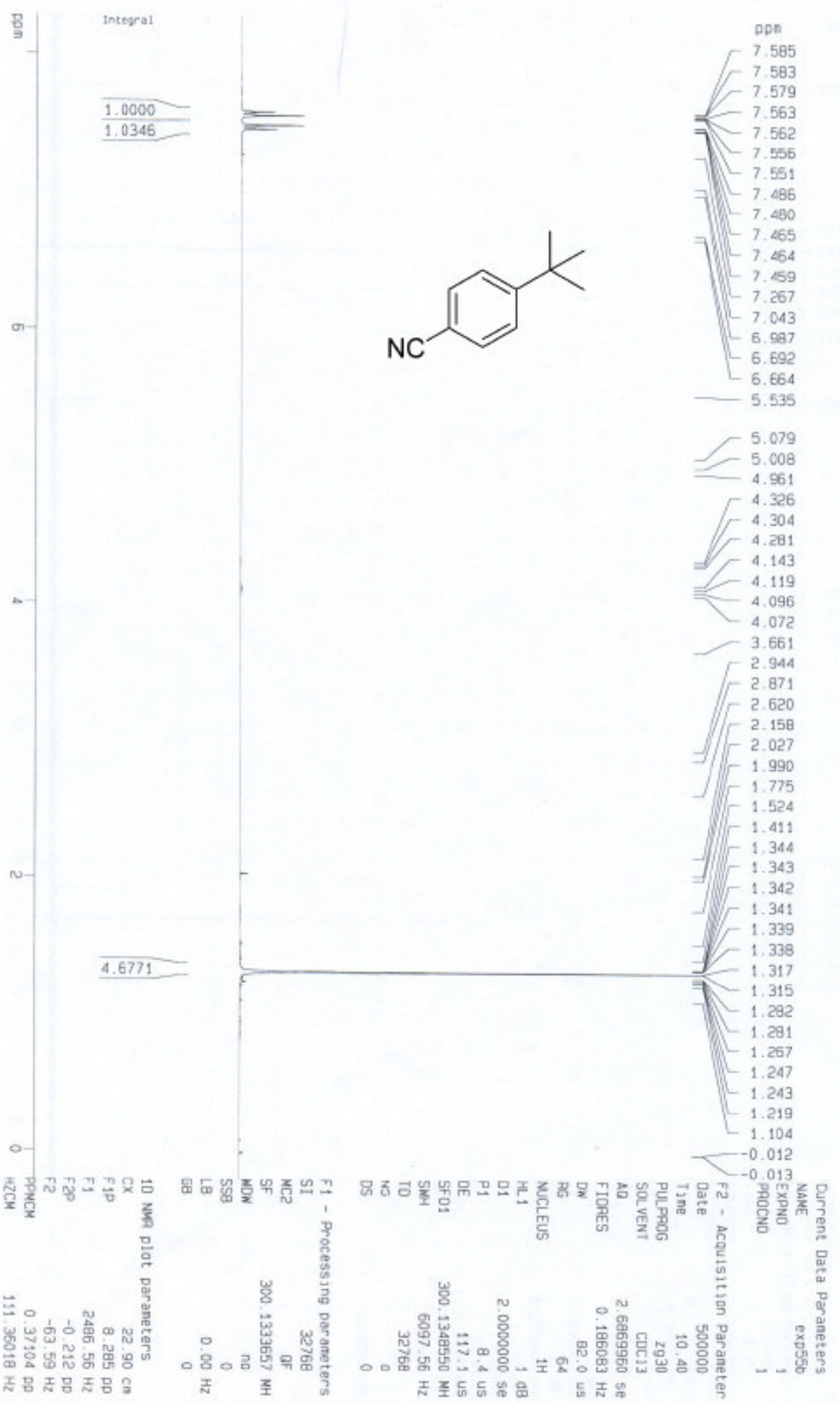




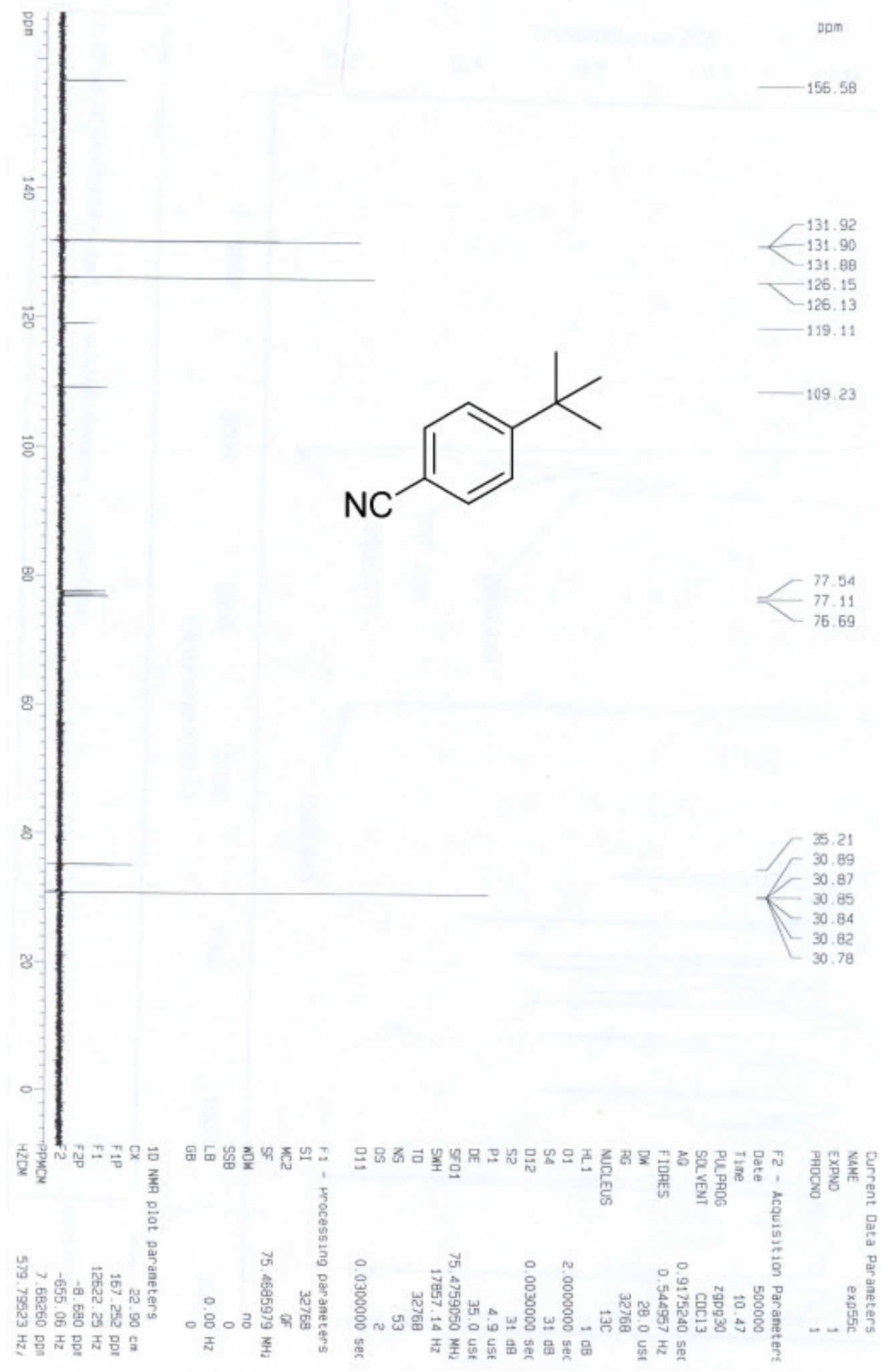




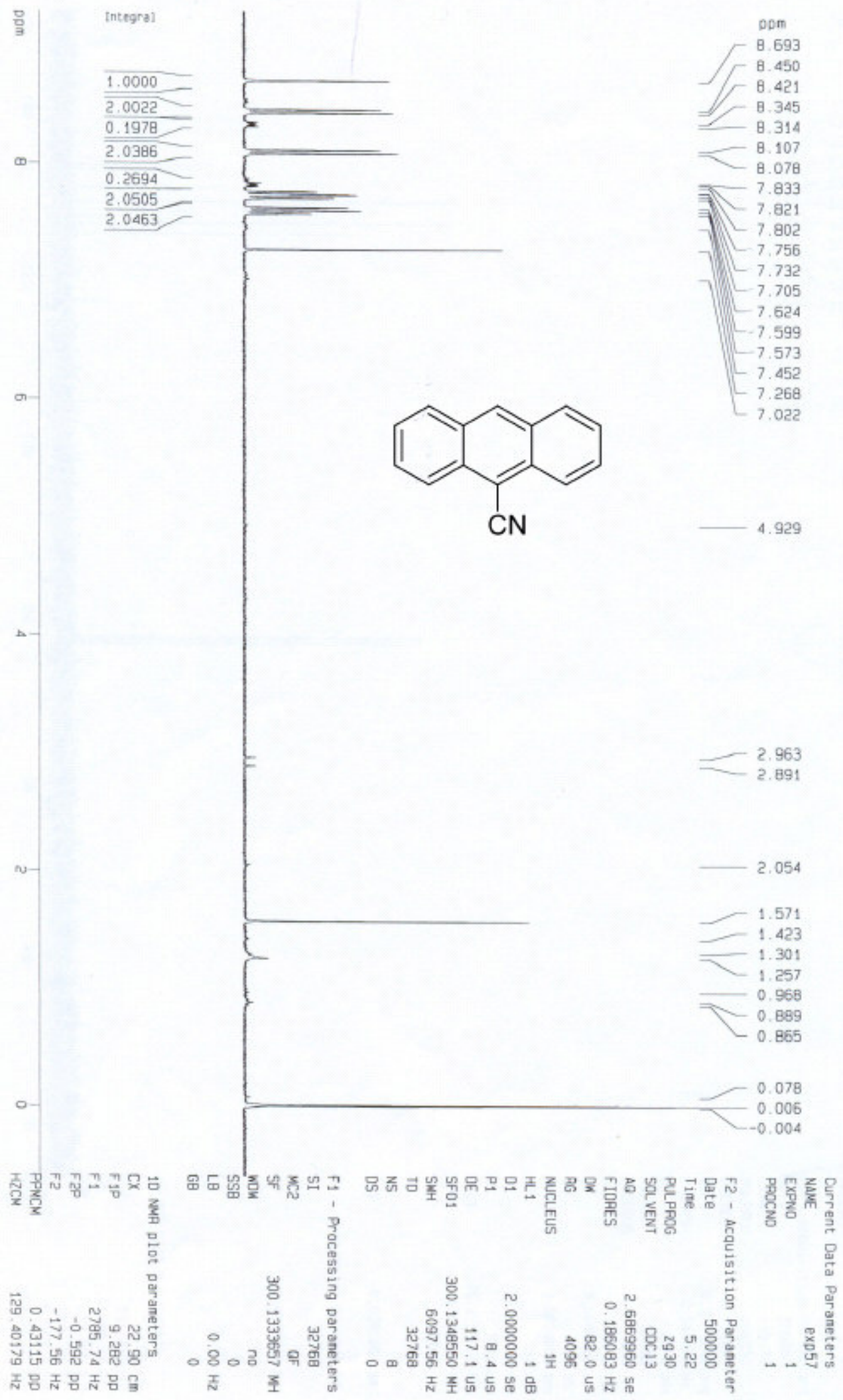




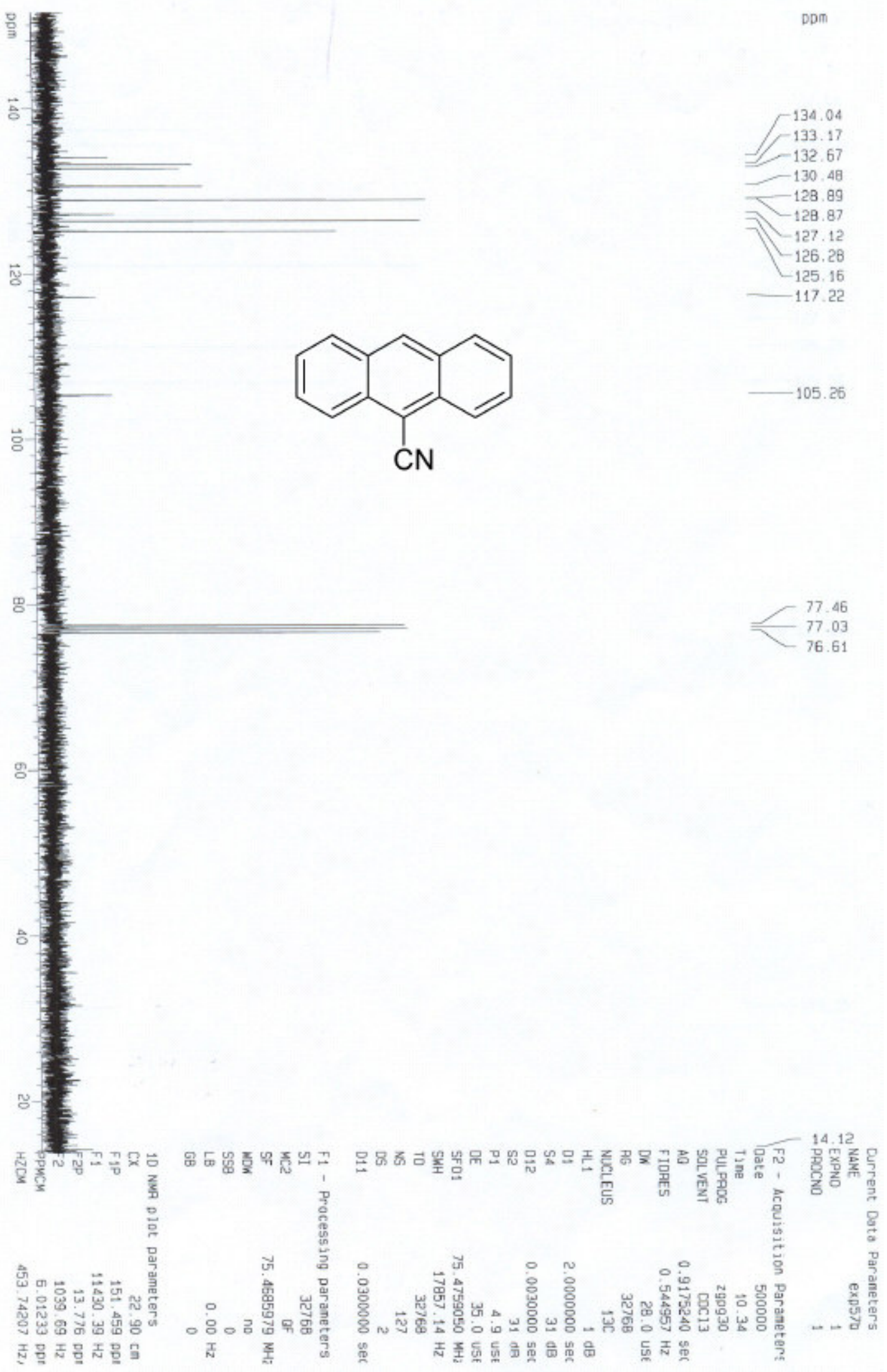




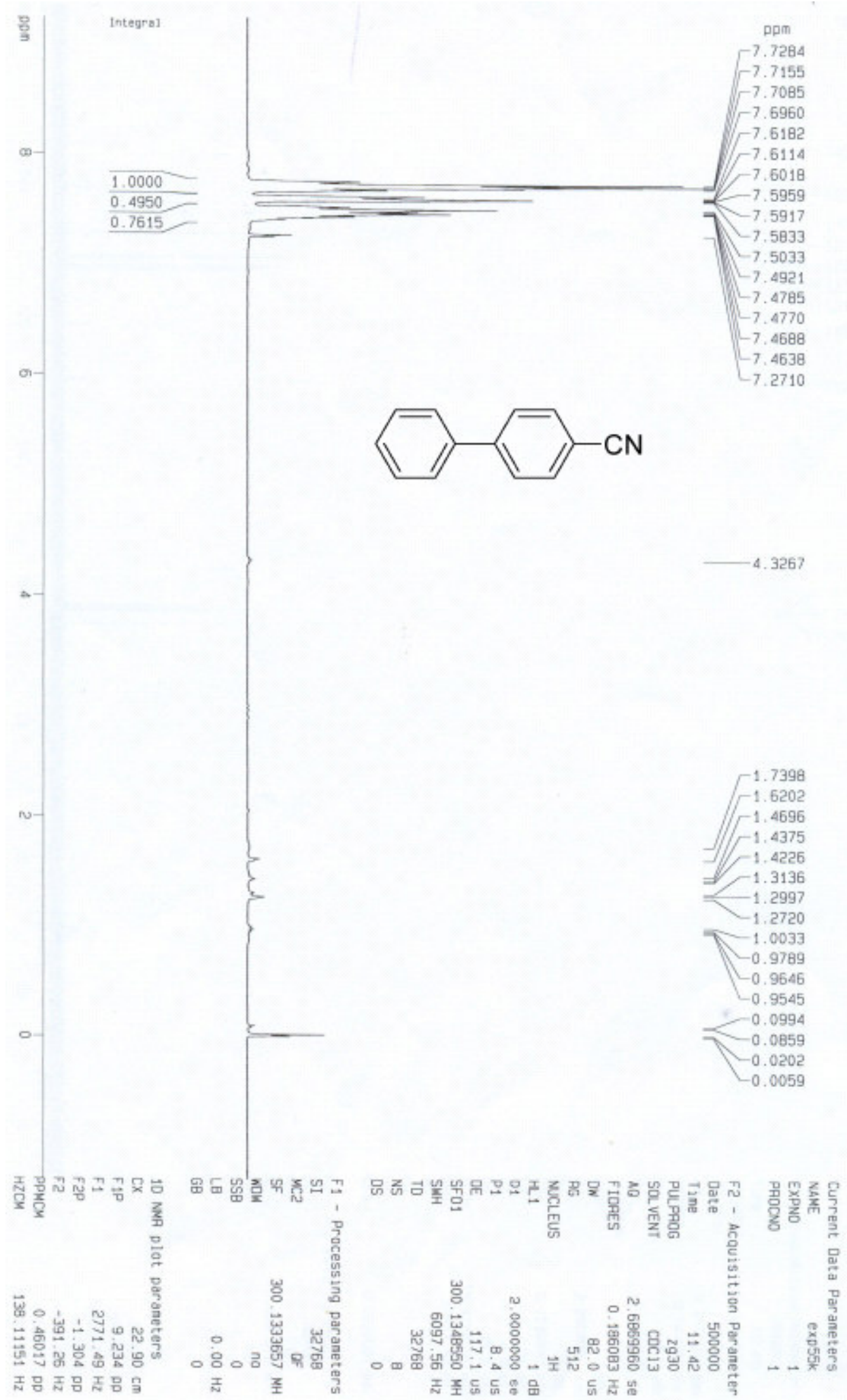




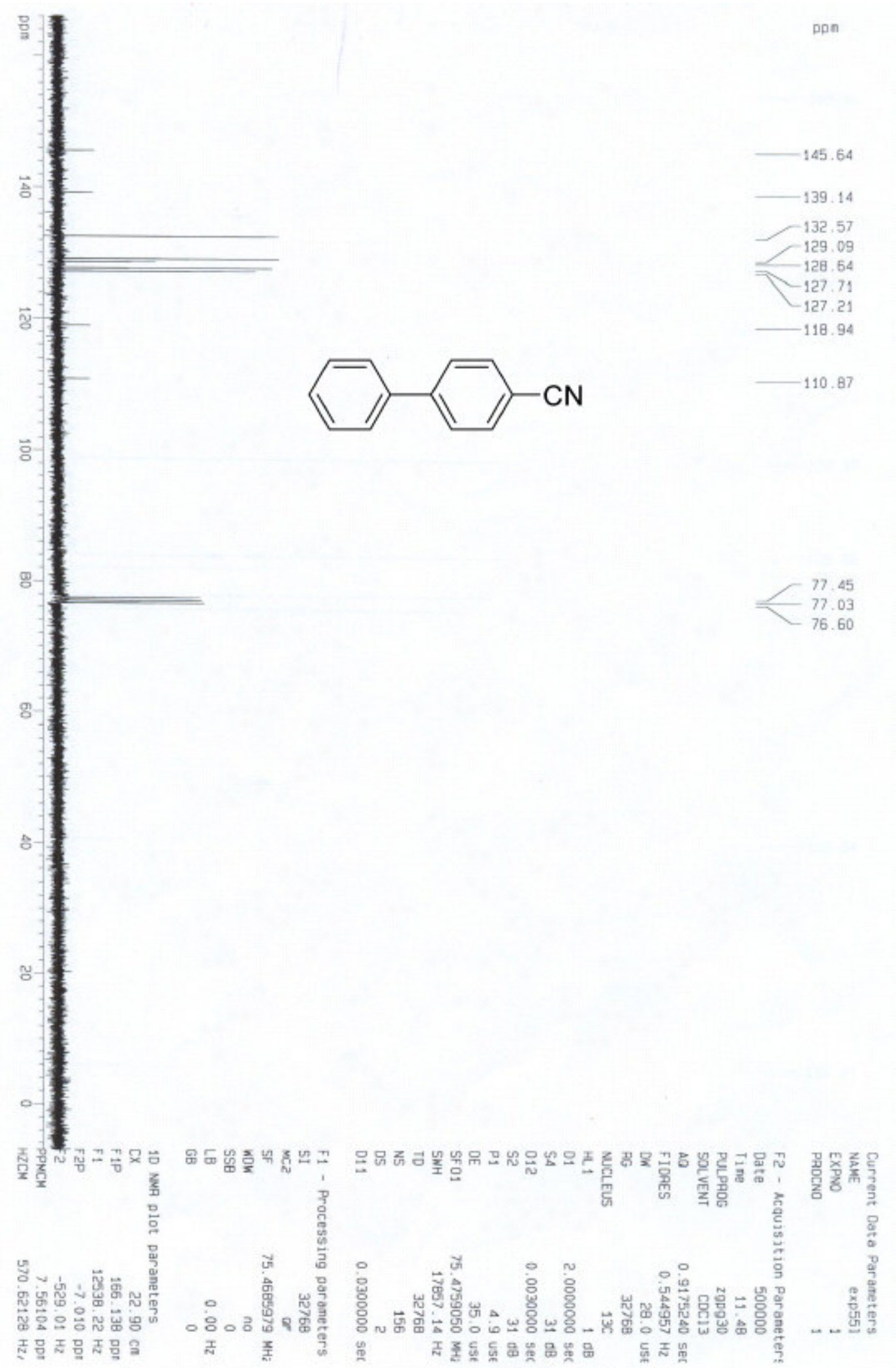




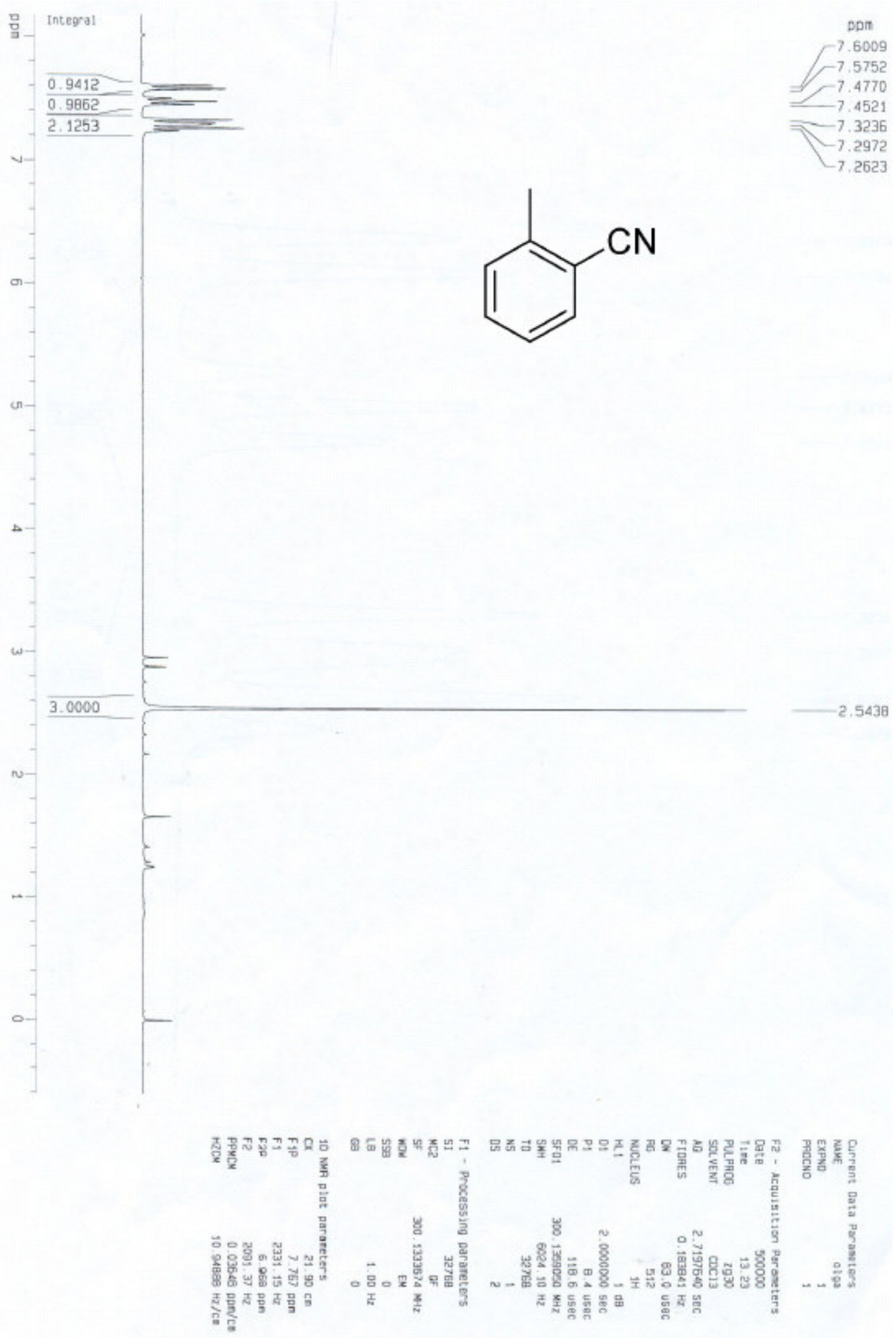




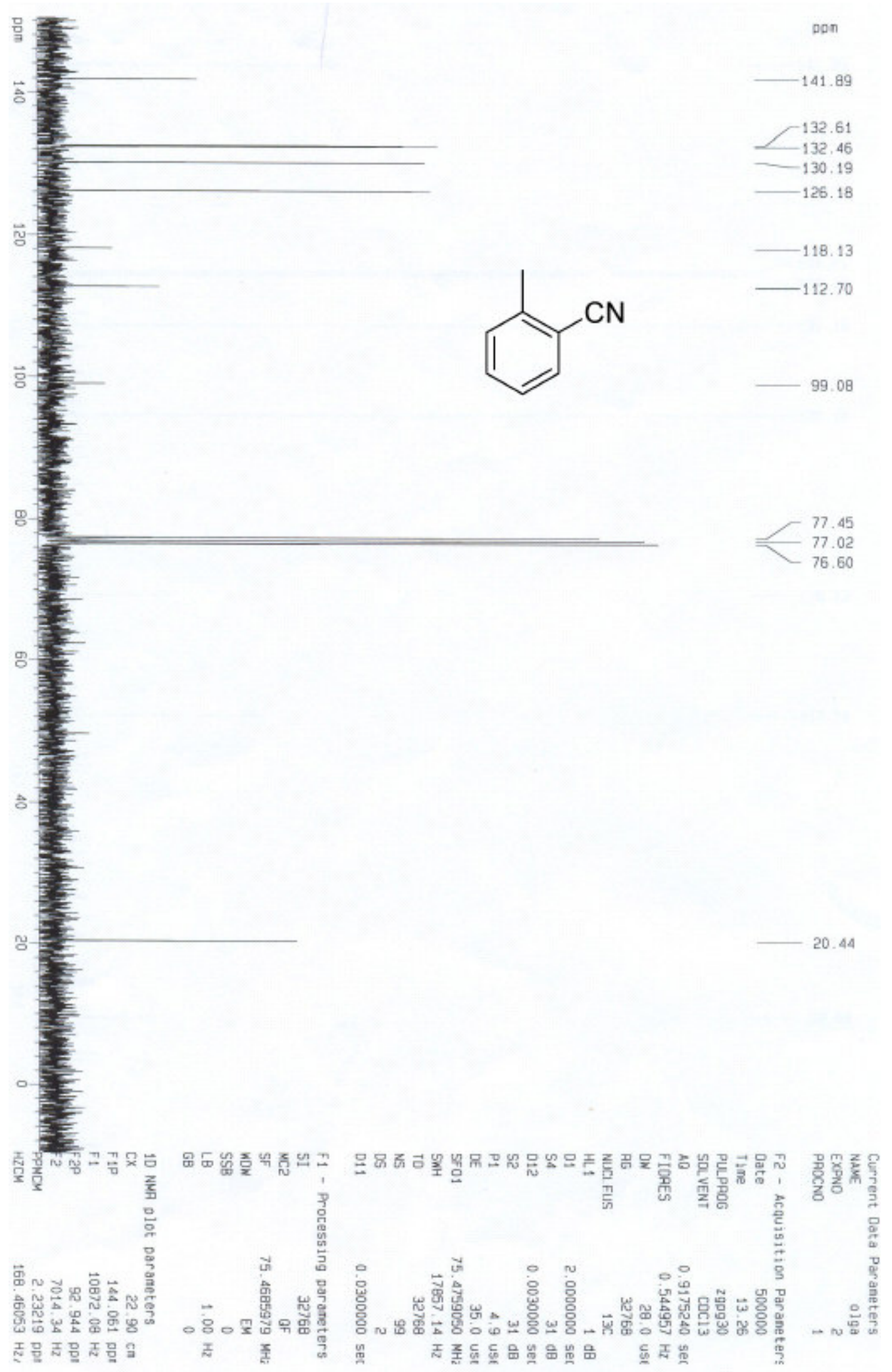




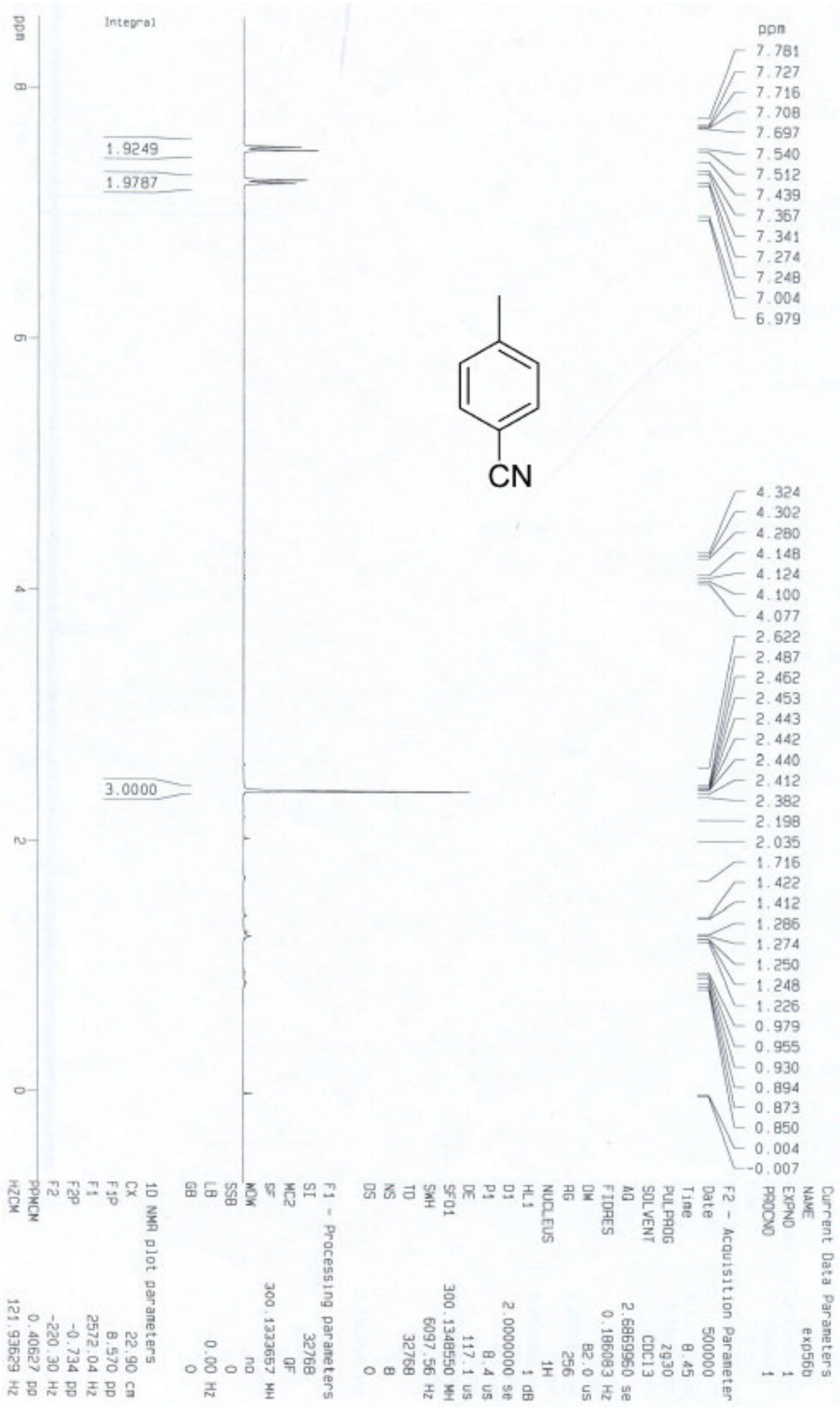




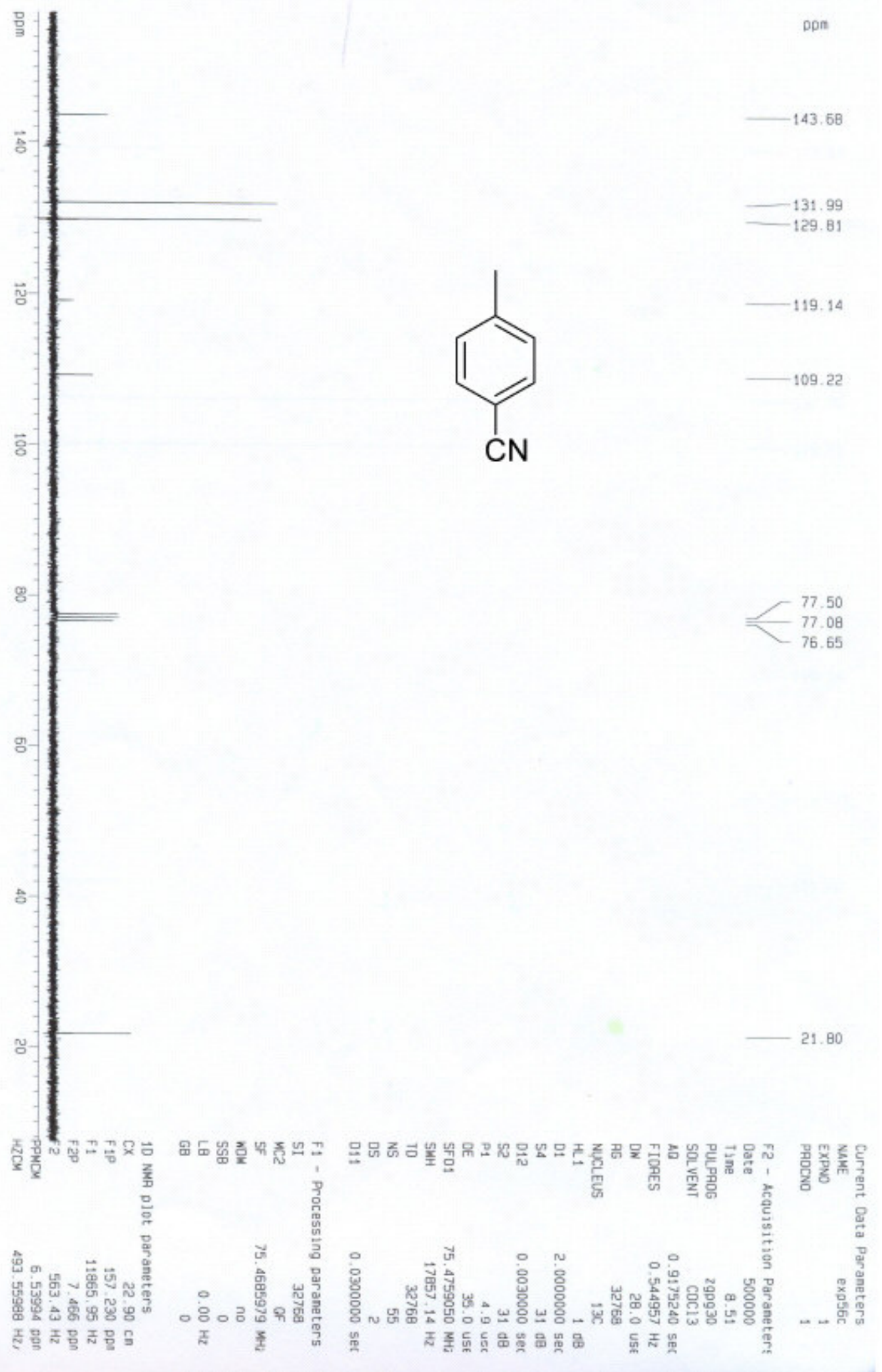




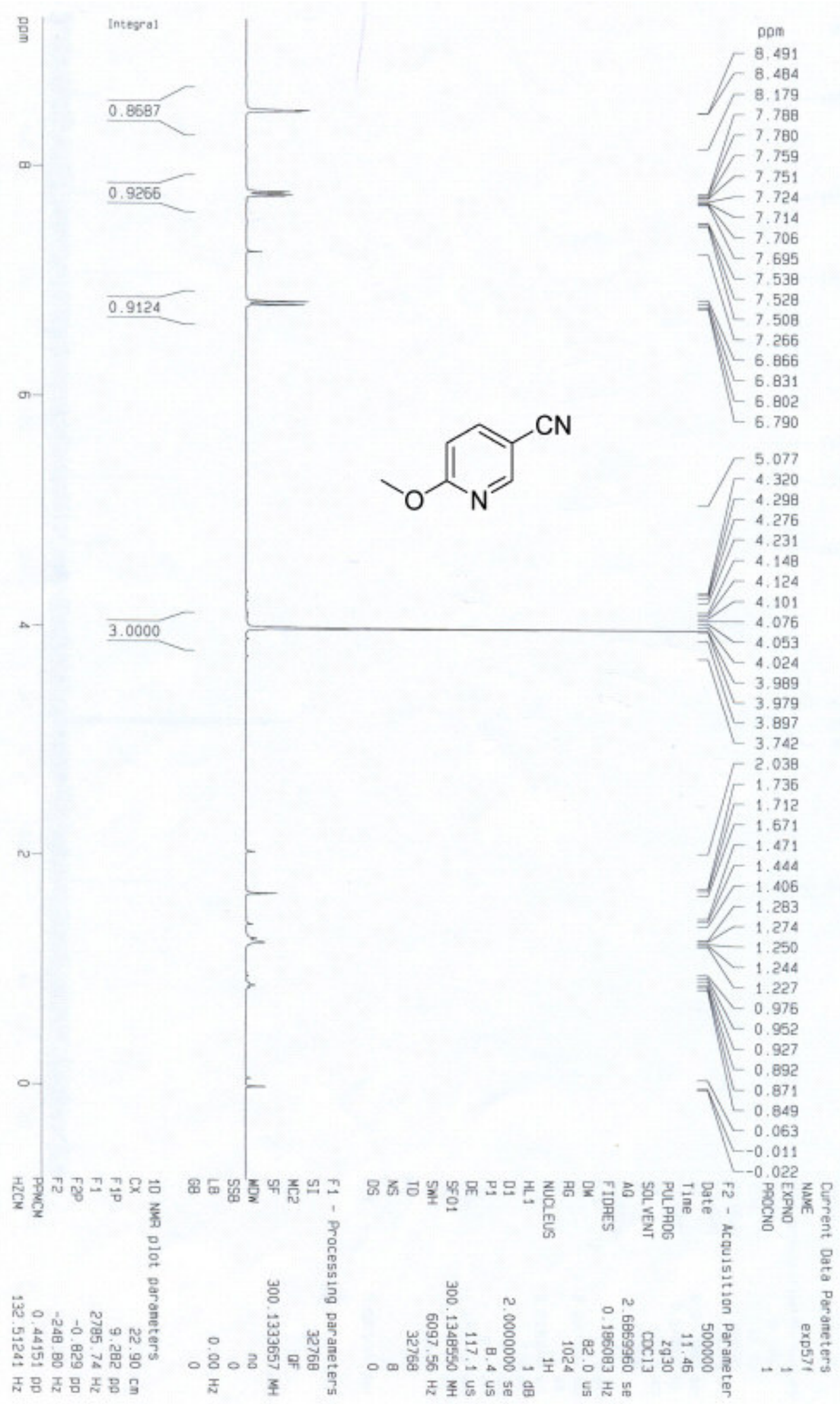




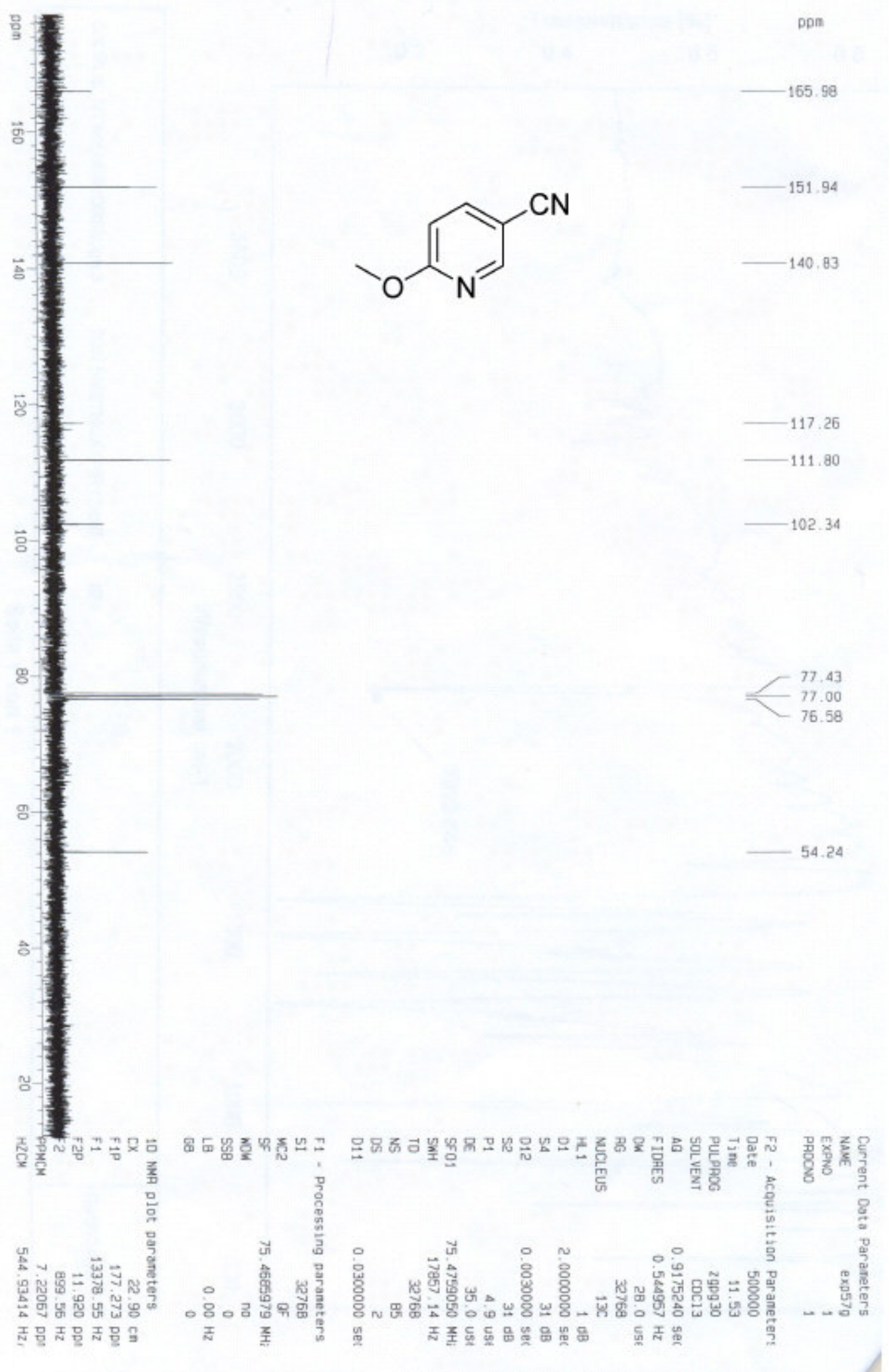




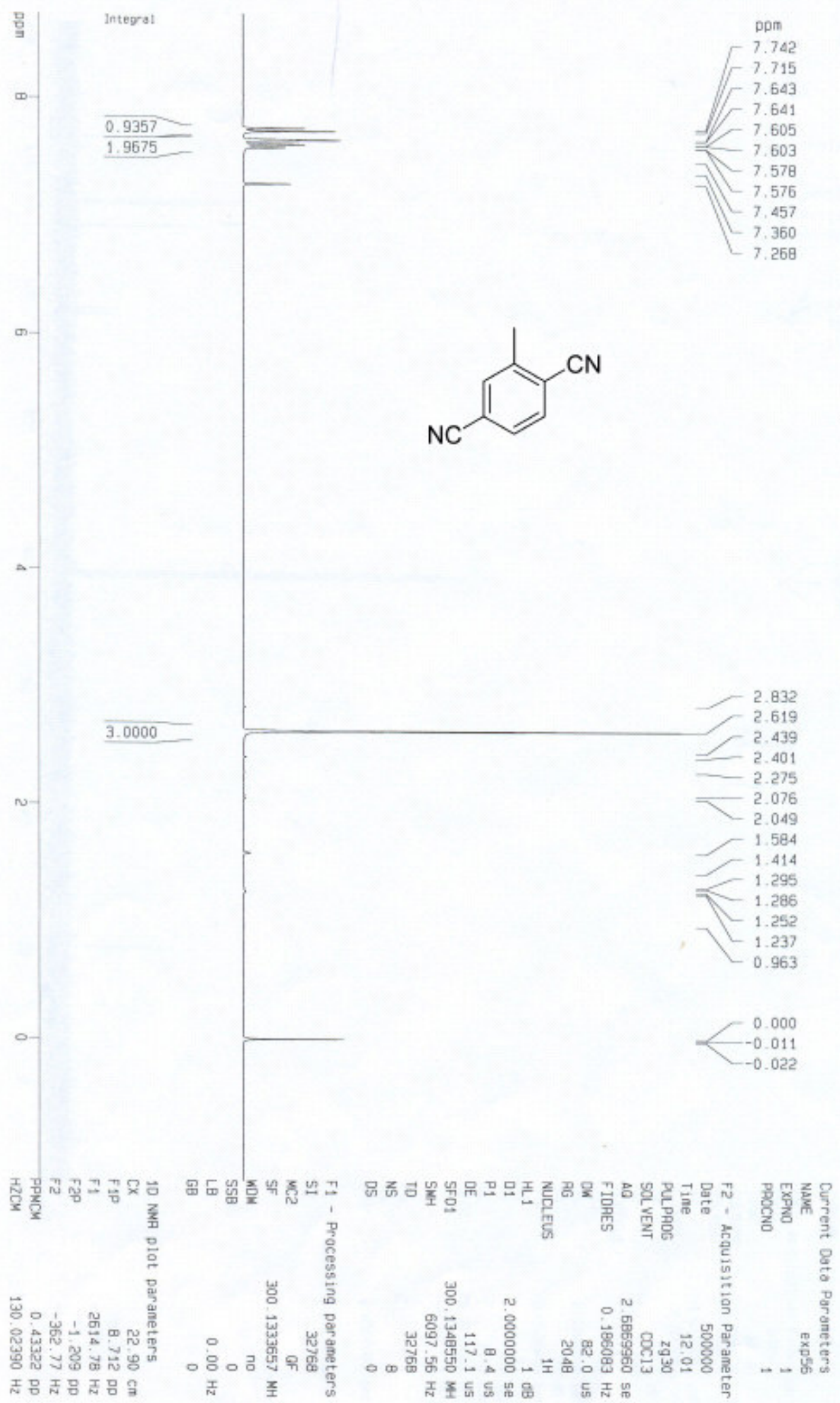




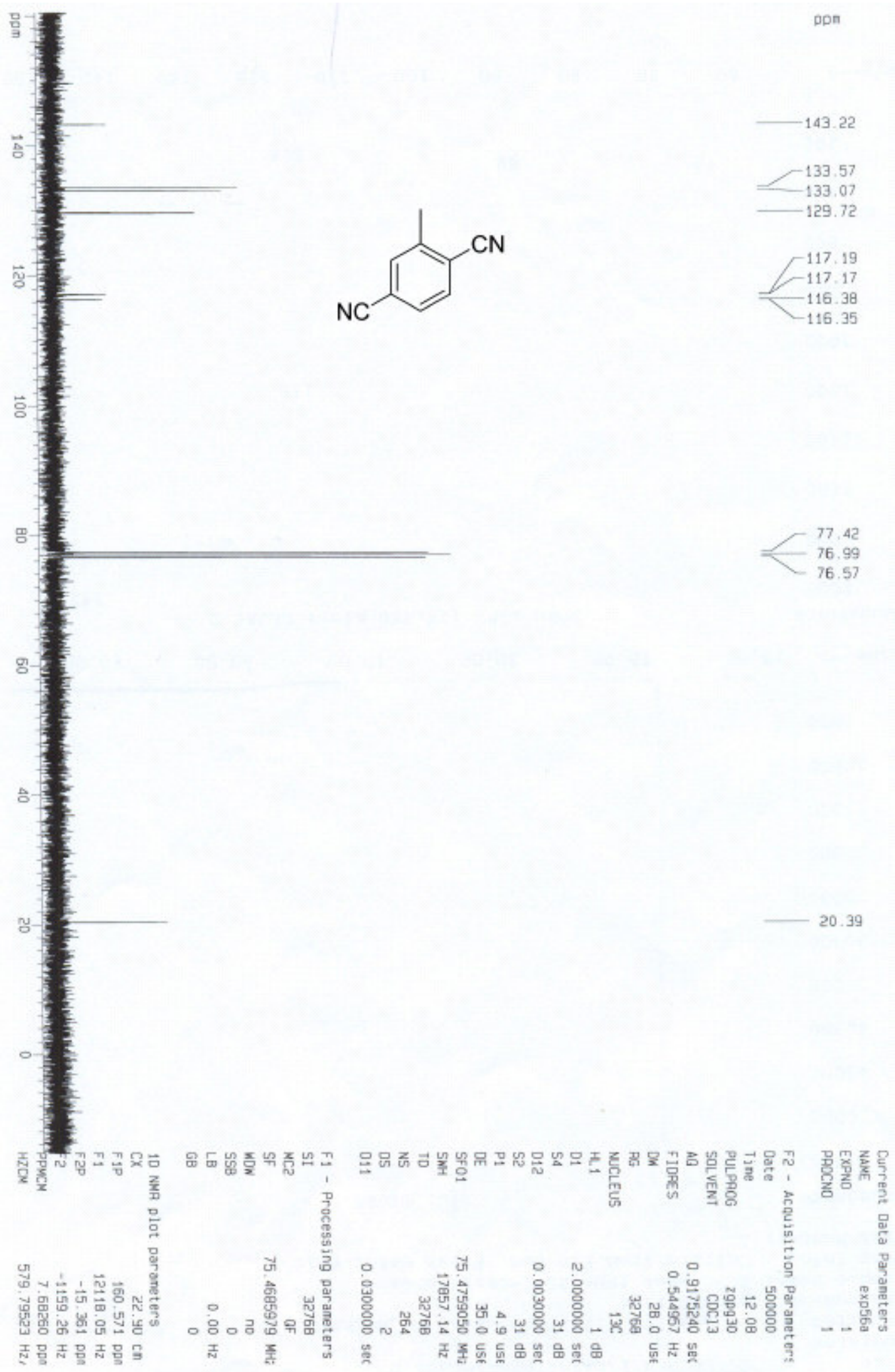

